\title{
Conformal 3D planned radiotherapy for pelvic lymphoceles following surgery for urological cancer: A case study
}

\author{
STEFAN JANSSEN $^{1,2}$, LUKAS KÄSMANN ${ }^{2}$, ROBERT CEGLA $^{1}$ and DIRK RADES ${ }^{2}$ \\ ${ }^{1}$ Private Practice of Radiation Oncology, D-30161 Hannover; \\ ${ }^{2}$ Department of Radiation Oncology, University of Lübeck, D-23538 Lübeck, Germany
}

Received March 10, 2015; Accepted May 25, 2016

DOI: $10.3892 / \mathrm{mco} .2016 .933$

\begin{abstract}
The aim of the present study was to evaluate the outcome and toxicity of 3D conformal radiotherapy (RT) for persistent lymphoceles following surgery for urological cancer. A total of 6 patients with bladder $(n=1)$ and prostate cancer $(n=5)$, with persistent lymphoceles following surgery for a primary tumor were treated with total doses of 10-12 Gy (1 Gy single dose) after computed tomography (CT) based 3D planning in order to suspend secretion. No acute or chronic toxicities were observed. In 5 patients, secretion of lymph fluid resolved after RT and in 1 patient RT had no effect. After a mean follow-up of 21 months (range, 5-47 months), no patient suffered from any symptoms concerning his former lymphoceles. This is the first analysis, to the best of our knowledge, to evaluate a homogenous patient collective of urological cancer patients with persistent lymphoceles after surgery for the initial tumor. RT to lymphoceles in urological cancer patient is effective, very well-tolerated and should be offered to patients with persistent secretion following drainage.
\end{abstract}

\section{Introduction}

Lymphoceles are an abnormal collection of lymphatic fluid occurring following surgery. They can cause abdominal pain, deep vein thrombosis or lower urinary tract problems (1). Incidence, for example following open radical prostatectomy with pelvic lymph node dissection, varies in the literature between 3 and 14\% (2). While treatment options encompass percutaneous aspiration with or without instillation of sclerosing agents and laparoscopic marsupialization, no standard is defined $(1,3)$. Very few papers have focussed on radiotherapy (RT) as an alternative treatment option using different doses and techniques (4-7). The aim of the present analysis was to evaluate outcome and toxicity of a homogenous treatment

Correspondence to: Dr Stefan Janssen, Private Practice of Radiation Oncology, 10 Rundestr, D-30161 Hannover, Germany E-mail: s.janssen@strahlentherapie.de

Key words: radiotherapy, urological cancer, postoperative lymphoceles schedule and modern technique for patients with postoperative persistent lymphoceles in the setting of malignant urological disease.

\section{Case study}

Following radical prostatectomy/cystectomy with lymphadenectomy, 6 patients developed a pelvic lymphocele, which persisted after percutaneous drainage. Each patient (seven treatment volumes in total) was treated with external beam RT using 3D planned treatment with virtual simulation after planning CT. Clinical target volume (CTV) was contoured, including the entire lymphocele with a margin of 1-2 cm, creating the planning target volume (PTV). Photons of 6 and 18MV energy were selected and 3-5 coplanar fields were used (Fig. 1). Of the patients, 1 patient was simultaneously irradiated to the prostate bed (66 Gy; 2 Gy single dose). Patient and treatment-associated parameters are summarized in Table I.

No acute toxicities or chronic side effects were observed. Of the 6 patients, 5 patients exhibited secretion of lymph fluid, which was resolved after RT (Table I). In 4 patients, secretion was already reduced during RT, while 1 patient responded time-delayed without further therapy ( 8 weeks after RT) (Table I). In 1 patient, RT caused no effect, making a marsupialization necessary. Following a mean follow-up of 21 months (range, 5-47 months), no patient suffered from any symptoms concerning his former lymphoceles. All drainages could be removed.

\section{Discussion}

Persistent lymphoceles are a common problem following pelvic surgery, particularly when lymphadenectomy is performed (3). Different approaches exist for the treatment of persistent lymphoceles but no standard is defined.

In previous literature, there are four studies exclusively focusing on RT for persistent lymphoceles $(4-6,8)$. From 1989-1998, Neu et al (4) treated 29 patients after vascular surgery with a single daily dose of 1 Gy up to 3-12 Gy. The majority of patients were treated with electrons (7-18 MeV) (4). Also, following vascular surgery, Dietl et al (6) treated 28 patients for inguinal lymphorrhea with single doses of 2-3 Gy up to 8-15 Gy. An orthovoltage of 120-300 KV 


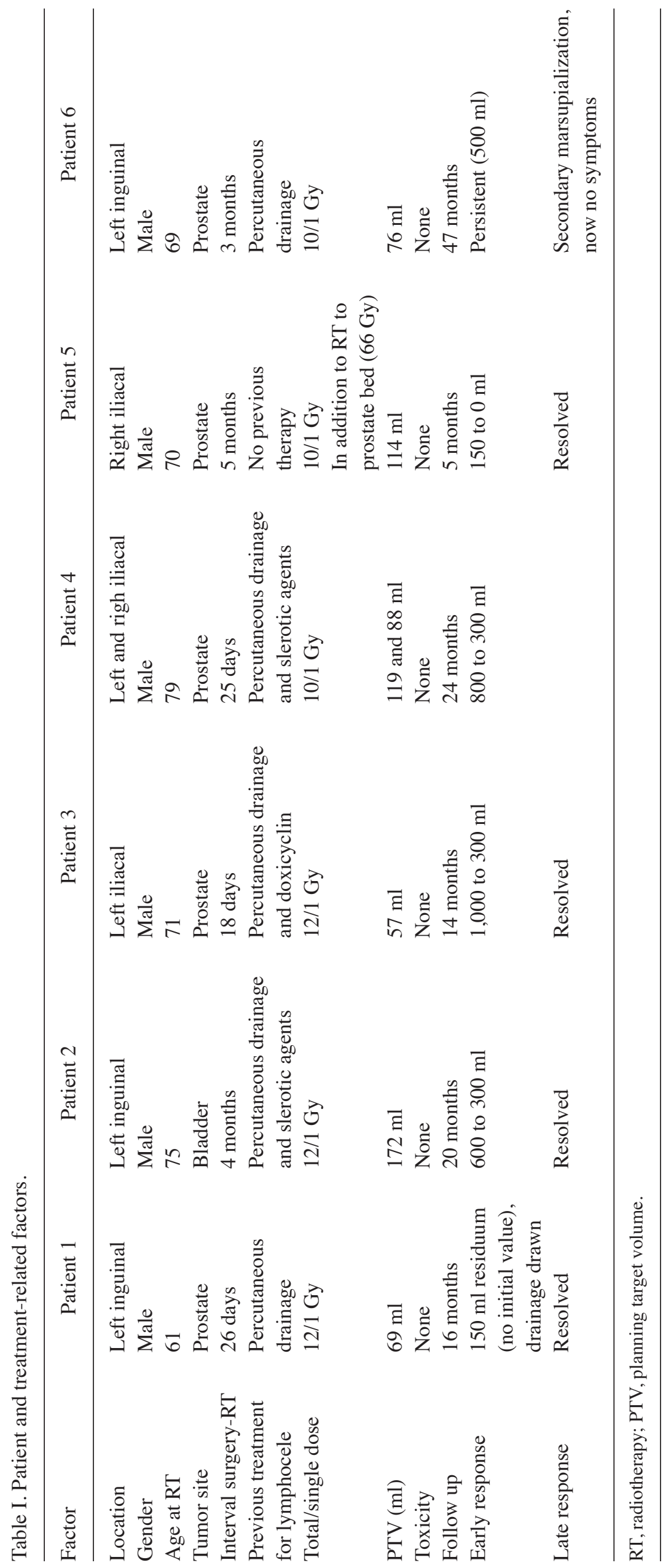




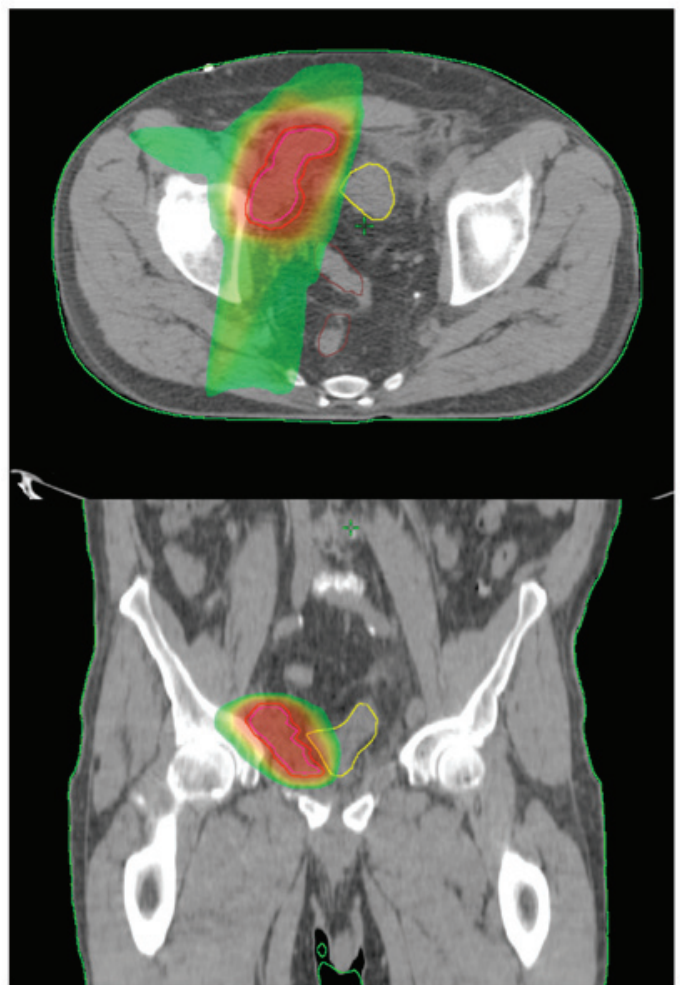

Figure 1. 3D conformal treatment plan for right inguinal lymphocele. Color-wash illustration showing dose distribution.

energy was used (6). The study group of Mayer et al (5) treated patients with fistulas after different, mostly vascular surgeries in different localizations with orthovoltage or electrons in majority. The single doses ranged from 0.3-2.0 Gy with total doses of 1-12 Gy (5). Previously, Jereczek-Fossa et al (8) showed RT after prostatectomy in the presence of asymptomatic lymphoceles to be feasible for integrating the lymphocele into the treatment plan of the prostate fossa with maximum doses to the lymphoceles of 5.7-73.3 Gy (8). Taken together, patient collectives of the above mentioned retrospective studies are heterogenous in localization, treatment technique, single and total doses, and primary surgery.

In the present study patients with urological cancer, primarily prostate cancer, were treated for inguinal/iliacal lymphoceles with a homogenous treatment schedule. Contrasting to the other study groups, a more precise RT technique was applied. Prior to the initiation of the treatment, a planning CT was performed, on which the planning target volume (lymphocele) was contoured. Afterwards a 3D treatment plan was generated. This allowed a more precise and conformal dose coverage of the target volume and sparing of the organs at risk compared with clinical approaches. In line with this, no acute or late side effects were observed.

The response rates (suspended secretion or removal of drains) varied between 76 and $93 \%$ in the literature, and compare well with the present findings (83\%).

A limitation of the present retrospective case series is without any doubt the small number of patients. Nevertheless, in the absence of prospective studies, the present case series is the first, to the best of our knowledge, to show the effectiveness and safety of modern RT to persistent lymphoceles in patients with urological cancer.

In conclusion, 3D conformal RT for persistent pelvic lymphoceles in patients with urological cancer is effective and very well-tolerated. Urological surgeons must be aware of this treatment option, particularly following the failure of other treatment modalities.

\section{References}

1. Raheem OA, Bazzi WM, Parsons JK and Kane CJ: Management of pelvic lymphoceles following robot-assisted laparoscopic radical prostatectomy. Urol Ann 4: 111-114, 2012.

2. MuschM,KleveckaV,RoggenbuckUandKroepflD:Complications of pelvic lymphadenectomy in 1,380 patients undergoing radical retropubic prostatectomy between 1993 and 2006. J Urol 179: 923-928; discussion 928-929, 2008.

3. Anheuser P, Treiyer A, Stark E, Haben B and Steffens JA: Lymphoceles after radical retropubic prostatectomy. A treatment algorithm. Urologe 49: 832-836, 2010 (In German).

4. Neu B, Gauss G, Haase W, Dentz J and Husfeldt KJ: Radiotherapy of lymphatic fistula and lymphocele. Strahlenther Onkol 176: 9-15, 2000 (In German).

5. Mayer R, Sminia P, McBride WH, Stranzl H, Prettenhofer U, Fruhwirth J and Poschauko J: Lymphatic fistulas: Obliteration by low-dose radiotherapy. Strahlenther Onkol 181: 660-664, 2005.

6. Dietl B, Pfister K, Aufschläger C and Kasprzak P: Radiotherapy of inguinal lymphorrhea after vascular surgery. A retrospective analysis. Strahlenther Onkol 181: 396-400, 2005.

7. Radiotherapy in benign disorders: Evidence-based guidelines of the German Association of Radiooncologists. (DEGRO) Version 1.0, 2013.

8. Jereczek-Fossa BA, Colangione SP, Fodor C, Russo S, Cambria R, Zerini D, Bonora M, Cecconi A, Vischioni B, Vavassori A, et al: Radiotherapy in prostate cancer patients with pelvic lymphocele after surgery: Clinical and dosimetric data of 30 patients. Clin Genitourin Cancer 13: e223-e228, 2015. 